\title{
Influência da modificação do teste de sentar e alcançar sobre o indicador de flexibilidade em diferentes faixas etárias
}

\author{
Influence of the modified Sit-and-Reach Test in flexibility of different age \\ groups
}

\author{
Ewertton de Souza Bezerra1,2,3*, Sandro Lemos Martins², Thalita Batista Leite ${ }^{2}$, Kayo \\ Douglas Vieira Paladino1, Mateus Rossato ${ }^{1,3}$, Roberto Simão ${ }^{2}$
}

ARTIGO ORIGINAL | ORIGINALARTICLE

\begin{abstract}
RESUMO
O objetivo do presente estudo foi comparar a influência do Teste de Sentar e Alcançar (TSA) modificado com e sem aquecimento sobre o score final obtido por diferentes faixas etárias de homens e mulheres fisicamente ativos. A amostra foi composta por 412 sujeitos, sendo 321 do gênero feminino $(45 \pm 14,09$ anos; $66 \pm 12,23 \mathrm{Kg} ; 1,55 \pm 0,05 \mathrm{~m}$ ) e 91 do gênero masculino (42 $\pm 15,75$ anos; 74,6 $\pm 12,41 \mathrm{Kg} ; 1,65$ $\pm 0,07 \mathrm{~m})$. Todos os indivíduos realizaram o TSA modificado e convencional, com e sem aquecimento. O TSA modificado apresentou resultados significativos quando comparado à forma convencional, em ambos os gêneros avaliados $(\mathrm{p}=0,0001)$. Em relação à condição para realizar o teste (com e sem aquecimento), o protocolo com aquecimento foi superior ao protocolo sem aquecimento, em ambos os gêneros, para o TSA modificado e convencional $(p=0,0001)$. Como conclusão, para avaliar a flexibilidade de membros inferiores, seria interessante utilizar um TSA adaptado, a fim de minimizar uma possível influência no score obtido no final do teste.

Palavras-chave: alongamento, flexibilidade, teste de sentar e alcançar.
\end{abstract}

ABSTRACT

The aim of this study was to compare the influence of the modified Sit-and-Reach test (MSR) with or without warm-up on the final score obtained by groups of men and women of different ages, physically actives. The sample consisted of 412 subjects, 321 females $(45 \pm 14.09$ years, $66 \pm 12.23 \mathrm{~kg}, 1.55 \pm$ $0.05 \mathrm{~m}$ ) and 91 males ( $42 \pm 15.75$ years; $74,6 \pm 12.41 \mathrm{~kg}, 1.65 \pm 0.07 \mathrm{~m})$. All subjects underwent the MSR and the conventional Sit-and-Reach test (CSR), with or without warm up. The MSR showed significant results when compared to the CSR protocol, in both genders $(p=0.0001)$. Regarding the condition for testing (with or without warm up), the protocol with warm up showed better results than the protocol without warm up, for both genders, for the MSR and CSR ( $p=0.0001$ ). In conclusion, to evaluate the lower body flexibility, it would be interesting to use a MSR to minimize a possible influence in the score obtained at the test.

Keywords: stretching, flexibility, sit-and-reach test.

\footnotetext{
Artigo recebido a 05.06.2013; Aceite a 24.09.2014

${ }^{1}$ Laboratório de Estudo do Desempenho Humano - LEDEHU- Faculdade de Educação Física e Fisioterapia Universidade Federal do Amazonas, -AM-Brasil

${ }^{2}$ Laboratório de Treinamento de Força- Escola de Educação Física e Desportos - Universidade Federal do Rio de Janeiro - RJ - Brasil

${ }^{3}$ Laboratório de Biomecânica - Centro de Desportos - Universidade Federal de Santa Catarina-SC-Brasil

* Autor correspondente: Av. General Rodrigo Octávio, 6200, Coroado I, 69077-000, Manaus, Brasil E-mail: ewsbezerra@yahoo.com.br
} 


\section{INTRODUÇÃO}

A flexibilidade é uma variável importante para o desempenho físico, saúde e qualidade de vida (Garber et al., 2011). Diversos fatores podem influenciar os níveis de flexibilidade, tais como: gênero, condição física, idade e especificidade de treinamento (Garber et al., 2011; Liemohn, Sharpe, \& Wasserman, 1994; Mier \& Shapiro, 2013).

Existem diversos métodos para realizar a avaliação da flexibilidade, um deles é o Teste de Sentar e Alcançar (TSA). Este teste foi desenvolvido para medir a flexibilidade da coluna vertebral e parte posterior dos membros inferiores de indivíduos de todas as idades e ambos os gêneros (Wells \& Dillon, 1952). O TSA é um dos mais conhecidos e bem difundidos métodos para avaliar a flexibilidade em centros de treinamento e academias, principalmente pelo fato de ser um teste de fácil aplicação, baixo custo e curta duração.

Segundo ACSM (2006), antes de se administrar o TSA não se deve deixar de realizar um aquecimento aeróbico de intensidade leve a moderada ( 5 - 10 minutos) e aquecimento muscular adequado. A utilização do aquecimento, que antecede a realização de algum tipo de exercício físico, é uma prática comumente utilizada, tanto por praticantes de atividade física quanto atletas de alto rendimento e tem como objetivo melhorar a dinâmica de um músculo para que ele seja menos propenso a lesão e, preparar os indivíduos para as demandas do exercício (Woods, Bishop, \& Jones, 2007). Diversos estudos fazem uso do aquecimento prévio a realização do TSA, Porém a metodologia aplicada no aquecimento se torna conflitante, poucos estudos utilizam o aquecimento proposto pelo ACSM 2006 em sua forma original. Outros estudos utilizam apenas exercícios de flexibilidade ou apenas a caminhada o que dificulta a comparação dos resultados na questão da limitação na utilização ou não do aquecimento prévio ao TSA (Mayorga-Vega, Merino-Marban, \& Viciana, 2014). Esse aquecimento realizado previamente ao TSA, diferenças de proporções entre comprimento dos membros superiores e inferiores, mobilidade da coluna vertebral e mobilidade da cintura escapular (D. R. Hopkins \& Hoeger, 1992; Simoneau, 1998), são algumas das limitações do TSA que consequentemente podem alterar a classificação final do nível de flexibilidade do avaliado.

Hopkins e Hoeger (1992) comparam o TSA original, proposto por Wells e Dillon (1952) com o TSA modificado, proposto por Hopkins (1981), o qual tinha como objetivo estabelecer um ponto zero para cada indivíduo, a fim de eliminar as diferenças de comprimentos dos membros, antes da realização do teste. Foram verificadas diferenças significativas em ambos os protocolos para um mesmo grupo. Assim, a fim de eliminar qualquer viés de comprimento dos membros e fornecer uma pontuação precisa da flexibilidade do tronco e quadril, os autores recomendam que o TSA modificado seja utilizado. Além disso, ambos os protocolos (original e modificado) não se preocuparam com a possível influência dos músculos gastrocnêmios, o que também poderia influenciar o score final dos testes. Essa musculatura, quando se encontra encurtada, pode alterar o alcance máximo do indivíduo durante o teste, o que poderia subestimar o alcance máximo dos avaliados (Cardoso, Azevedo, Cassano, Kawano, \& Âmbar, 2007). Liemohn, Sharpe, e Wasserman (1994) sugeriram uma adaptação ao TSA original a fim de minimizar a influência dos músculos gastrocnêmios. Eles sugeriram uma adaptação na caixa, onde durante a realização do teste o pé não ficaria fixo e sim móvel; assim, diferente do TSA convencional, o indivíduo poderia manter o pé em dorsiflexão ou em flexão plantar.

Outra adaptação proposta por Cardoso, Azevedo, Cassano, Kawano, e Âmbar (2007), para reduzir uma possível influência dos músculos gastrocnêmios, é colocar uma abertura (porta) de $27 \mathrm{~cm}$ de altura x $27 \mathrm{~cm}$ de largura no local do apoio plantar, na caixa. Esta porta, quando aberta, possibilita eliminar a influência dos músculos gastrocnêmios 
durante o teste. Essa adaptação não foi utilizada em estudos anteriores, quando o objetivo era comparar o TSA original e o adaptado.

Sendo assim, o objetivo do presente estudo é: (a) comparar a influência do Teste de Sentar e Alcançar modificado (com abertura na caixa) realizado com e sem aquecimento prévio, sobre o score final obtido por diferentes faixas etárias de homens e mulheres fisicamente ativos; (b) propor tabelas percentis para facilitar a classificação de indivíduos nas diferentes faixa-etárias avaliadas.

\section{MÉTODO}

Este estudo tem caráter quase experimental, pois não apresenta grupo controle. A caracterização da amostra foi realizada através de medidas antropométricas (estatura, massa corporal e IMC). Para as medidas antropométricas, foi utilizada uma balança digital (Welmy ${ }^{\circledR}$, Brasil), calibrada em quilogramas e um estadiômetro (Sanny, Brasil), graduado em centímetros. O IMC foi calculado adotando-se como padrão de referência a equação do World Health Organization (1995). Em seguida, a realização do TSA foi feita na seguinte ordem: sem aquecimento (porta aberta e fechada) e com aquecimento (porta aberta e fechada).
Antes da coleta de dados, todos os indivíduos responderam ao questionário PARQ (Shephard, 1988) e foram informados sobre o procedimento experimental, de acordo com a declaração de Helsinki. O Projeto de Pesquisa foi submetido e aprovado pelo Comitê/Conselho de Ética em Pesquisa da Instituição (0121.0.115.000-10 9) e atendeu às "Normas Sobre Pesquisa Envolvendo Seres Humanos", de acordo com a resolução 196/96 do Conselho Nacional de Saúde, de 10 de Outubro de 1996.

\section{AMOSTRA}

O grupo de voluntários foi composto por 412 sujeitos, sendo 321 do gênero feminino $(45 \pm 14,09$ anos; $66 \pm 12,23 \mathrm{Kg} ; 1,55 \pm 0,05$ m) e 91 do gênero masculino $(42 \pm 15,75$ anos; $74,6 \pm 12,41 \mathrm{Kg} ; 1,65 \pm 0,07 \mathrm{~m})$. Os sujeitos faziam parte de três programas de atividade física desenvolvidos na cidade de Manaus no período de Novembro de 2010 a Abril de 2011. O tamanho da amostra coletada foi de 76 (58 mulheres e 18 homens) no Centro de Convivência do Mutirão, 235 (182 mulheres e 53 homens) no Centro de Convivência Padre Pedro Vignola e de 101 (81 mulheres e 20 homens) no Centro de Convivência do Idoso.

Tabela 1.

Distribuição da amostra em relação a população por sexo e centro de atividade física estudado.

\begin{tabular}{|c|c|c|c|c|c|c|c|c|}
\hline Local & População & $\begin{array}{c}\text { Frequência } \\
\text { Relativa (\%) }\end{array}$ & & Amostra & $\begin{array}{c}\text { Frequência } \\
\text { Relativa (\%) }\end{array}$ & & Amostra & $\begin{array}{c}\text { Frequência } \\
\text { Relativa (\%) }\end{array}$ \\
\hline 1 & 6849 & 14 & \multirow{4}{*}{$\frac{\mathscr{d}}{\stackrel{d}{ \pm}}$} & 58 & 70 & \multirow{4}{*}{ 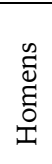 } & 18 & 30 \\
\hline 2 & 31329 & 62 & & 182 & 77 & & 53 & 23 \\
\hline 3 & 12342 & 24 & & 81 & 80 & & 20 & 22 \\
\hline Total & 50520 & 100 & & 321 & 78 & & 91 & 22 \\
\hline
\end{tabular}

Nota: 1) Centro de Convivência da Família do Mutirão; 2) Centro de Convivência da Família Padre Pedro Vignola; 3) Centro de Convivência do Idoso

A amostra foi definida com o objetivo de informar o número mínimo esperado de sujeitos da pesquisa, calculou-se por meio da fórmula de estimativa para proporções, considerando uma prevalência de $50 \%$ (p), nível de $95 \%$ de confiança e significância de $5 \%$ (d) de acordo com a equação:.

$$
n=\frac{Z^{2} \cdot \hat{p} \cdot \hat{q} \cdot N}{d^{2}(N-1)+Z^{2} \cdot \hat{p} \cdot \hat{q}}
$$

Após correção para população finita, uma amostra final de 412 indivíduos foi obtida. Visando resguardar a precisão, optou-se por majorar a amostra em $10 \%$ a fim de compensar eventuais perdas.

Como critérios de inclusão, foram adotados: a) ausência de relatos de alterações osteomioarticulares que impedissem a realização dos movimentos envolvidos no teste 
e, b) envolvimento em atividades de alongamento pelo menos duas vezes na semana a no mínimo seis meses, c) não praticar atividades físicas antes da realização dos procedimentos experimentais, para que a coleta dos dados não sofra alterações, d) não está em período gestacional.

As coletas de dados foram realizadas com intervalos de 24 horas, durante os período vespertino/noturno, pois este era o horário de maior fluxo de sujeitos nos centro de convivência da família. A coleta foi feita em uma sala climatizada em média com $25^{\circ} \mathrm{C}$.

\section{AQUECIMENTO}

O aquecimento foi aplicado especificamente para as musculaturas tóracolombar, isquiostibiais e tríceps sural. Para tal fim, dois exercícios de alongamento através do método dinâmico foram aplicados separadamente, durante o tempo cronometrado de um minuto. No primeiro exercício o sujeito na posição em pé realizou o movimento de flexão plantar e, no segundo exercício, também em pé, foi realizada flexão de quadril e coluna tóraco-lombar com inclinação do tronco em direção ao chão, juntamente com a extensão dos cotovelos,

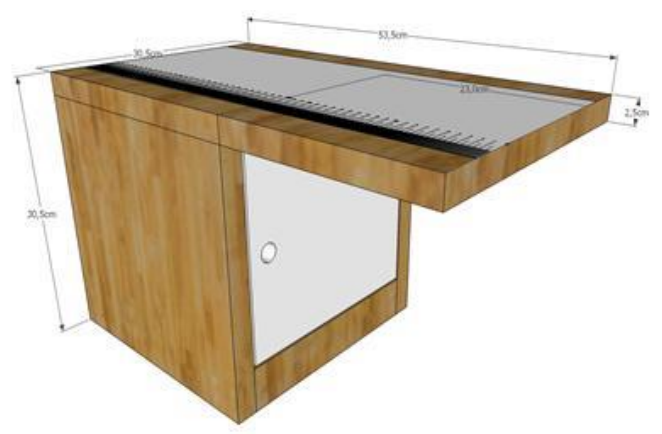

estando as mãos uma sobre a outra. Nos dois exercícios o sujeito voltava à posição de origem, realizando o ciclo do movimento até o fim do tempo determinado.

\section{PROCEDIMENTOS}

\section{Teste de Sentar e Alcançar}

O TSA foi realizado em uma caixa de madeira com as seguintes medidas $30,5 \mathrm{~cm} \mathrm{x}$ $30,5 \mathrm{~cm} \times 30,5 \mathrm{~cm}$, com um prolongamento de $23 \mathrm{~cm}$, para o apoio dos membros superiores dos sujeitos. Sobre a face superior da caixa e do prolongamento, havia uma escala métrica de $53,5 \mathrm{~cm}$ que permitiu determinar o alcance do indivíduo. No banco padrão, durante o teste, a planta dos pés do participante coincide com o $23^{\circ} \mathrm{cm}$ (vigésimo terceiro centímetro) da fita métrica. No local do apoio plantar na caixa foi construída uma abertura (porta) de $27 \mathrm{~cm}$ de altura x $27 \mathrm{~cm}$ de largura. Esta porta, quando aberta, possibilita eliminar a influência dos músculos gastrocnêmios durante o teste, fato que poderia subestimar o alcance máximo dos voluntários quando estes músculos encontram-se encurtados (Cardoso et al., 2007). Todas as medidas do instrumento são apresentadas na figura 2 .

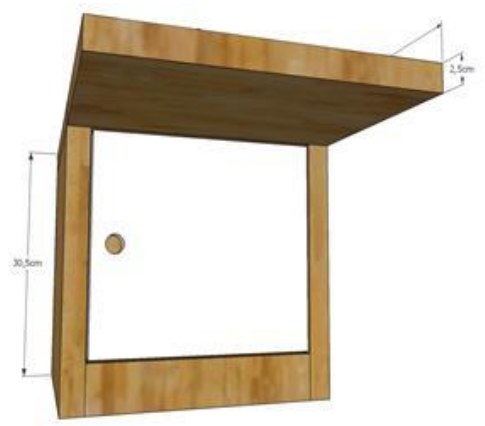

Figura 1. Instrumento para avaliação da flexibilidade, banco sentar e alcançar modificado, com as medidas em escala $(\mathrm{cm})$ e vista 3D.

Para o registro das duas condições experimentais, o indivíduo foi posicionado no instrumento descalço, com quadris em flexão, joelhos em extensão e a planta dos pés em contato com a parte frontal, na marca de 23 centímetros da escala. Foi solicitado que o participante realizasse inspiração profunda e, durante a expiração, inclinasse o tronco para frente com a intenção de alcançar a distância máxima possível com a ponta dos dedos. Para um controle sobre possíveis deslocamentos laterais dos indivíduos durante o teste, os membros superiores foram alinhados com a fita métrica do banco, com uma mão sobreposta à outra (dedo médio da mão direita sobre o da esquerda). Durante a realização do 
TSA o avaliador colocou as mãos sobre os joelhos do avaliado para que o mesmo não realizasse o movimento de flexão do joelho. $\mathrm{Na}$ forma de aplicação do TSA com a porta aberta, o sujeito realizava o mesmo padrão de movimento descrito anteriormente, com exceção do apoio frontal dos pés que era feito apenas pelo calcanhar, ficando a região do antepé e médiopé livres. Em ambas as condições e formas de aplicação do teste, o resultado foi observado na fita métrica fixada na parte superior da caixa, em três tentativas, ficando como resultado final a de maior valor. $\mathrm{O}$ resultado foi expresso em centímetros com uma casa decimal.

\section{ANÁLISE ESTATÍSTICA}

Os valores observados para as duas situações foram descritos pela média e desvio padrão. Inicialmente foi testada a normalidade dos resultados por gênero através do teste de Kolmorov-Smirnov ( $p>0,05)$. Como todas as variáveis apresentaram-se paramétricas, um Test T-student pareado foi aplicado para analisar as condições com e sem aquecimento nas duas formas de aplicação do TSA (porta aberta e fechada) em todas as faixas etárias analisadas para ambos os gêneros. Uma distribuição de frequência (percentil) foi utilizada para classificação em cada faixa etária. O valor alfa de 0,05 foi adotado e todo o tratamento estatístico foi realizado no pacote SPSS 17 for Windows.

\section{RESULTADOS}

O TSA modificado apresentou melhores resultados quando comparado à forma convencional, com diferença significativa em ambos os gêneros avaliados $(p=0,0001)$. Em relação à condição para realizar o teste (com e sem aquecimento), o protocolo com aquecimento foi superior ao protocolo sem aquecimento para ambos os gêneros, para o TSA modificado e convencional $(p=0,0001)$. Todos os resultados apresentados não sofreram modificação em função da faixa etária avaliada (Tabela 2).

Tabela 2

Resultados (média, desvio padrão) das comparações entre a situação convencional (C) e adaptada (A) do Teste Sentar e Alcançar nas condiçôes sem (SA) e com aquecimento (CA) por faixa-etária para o gênero feminino $(F)$ e masculino $(M)$.

\begin{tabular}{|c|c|c|c|c|}
\hline \multirow{2}{*}{$\begin{array}{c}\text { Teste } \\
\mathrm{F}\end{array}$} & \multicolumn{2}{|c|}{ Convencional $(\mathrm{cm})$} & \multicolumn{2}{|c|}{ Adaptado $(\mathrm{cm})$} \\
\hline & SA & $\mathrm{CA}$ & SA & CA \\
\hline $18-29$ & $28.02 \pm 6.46$ & $30.80 \pm 6.33^{b}$ & $30.55 \pm 6.48^{a}$ & $32.44 \pm 5.94^{\mathrm{ab}}$ \\
\hline $30-39$ & $31.12 \pm 5.52$ & $28.68 \pm 6.17^{b}$ & $32.79 \pm 5.40^{\mathrm{a}}$ & $30.87 \pm 5.90^{\mathrm{ab}}$ \\
\hline $40-49$ & $31.16 \pm 7.24$ & $28.70 \pm 7.33^{b}$ & $32.10 \pm 7.16^{\mathrm{a}}$ & $30.40 \pm 7.25^{\mathrm{ab}}$ \\
\hline $50-60$ & $30.43 \pm 7.28$ & $28.17 \pm 7.51^{b}$ & $31.82 \pm 6.89^{a}$ & $29.70 \pm 7.55^{\mathrm{ab}}$ \\
\hline$>60$ & $30.30 \pm 7.51$ & $27.98 \pm 7.98^{b}$ & $31.40 \pm 7.85^{\mathrm{a}}$ & $29.88 \pm 7.98^{\mathrm{ab}}$ \\
\hline $\mathrm{M}$ & SA & $\mathrm{CA}$ & SA & $\mathrm{CA}$ \\
\hline $18-35$ & $33.99 \pm 5.61$ & $30.46 \pm 6.61^{b}$ & $34.99 \pm 5.56^{\mathrm{a}}$ & $32.55 \pm 6.07^{\mathrm{ab}}$ \\
\hline $36-50$ & $27.92 \pm 7.08$ & $25.87 \pm 6.67^{b}$ & $29.20 \pm 7.45^{\mathrm{a}}$ & $27.34 \pm 7.45^{\mathrm{ab}}$ \\
\hline$>50$ & $29.63 \pm 7.82$ & $26.86 \pm 7.43^{b}$ & $30.86 \pm 7.53^{a}$ & $28.89 \pm 7.58^{\mathrm{ab}}$ \\
\hline
\end{tabular}

a diferença significativa em relação ao convencional $(p<0.05)$;

${ }^{b}$ diferença significativa em relação à condição sem aquecimento $(p<0.05)$

A partir dos resultados encontrados foram criadas tabelas referenciais para ambos os gêneros, feminino (Tabela 3) e masculino (Tabela 4), baseadas em percentil para classificar os tipos de testes propostos (convencional e modificado). As tabelas foram propostas a partir da condição com aquecimento 
Tabela 3

Valores percentis da flexibilidade (cm) para o TSA, convencional (TC) e TSA modificado (TM) na condição com aquecimento para o gênero feminino.

\begin{tabular}{|c|c|c|c|c|c|c|c|c|c|c|c|}
\hline \multirow{2}{*}{ Percentil } & \multicolumn{2}{|c|}{$18-29$} & \multicolumn{2}{|c|}{$30-39$} & \multicolumn{2}{|c|}{$40-49$} & \multicolumn{2}{|c|}{$50-60$} & \multicolumn{2}{|c|}{$>60$} & \multirow{2}{*}{ Classificação } \\
\hline & $\mathrm{TC}$ & TM & $\mathrm{TC}$ & TM & $\mathrm{TC}$ & TM & $\mathrm{TC}$ & TM & $\mathrm{TC}$ & $\mathrm{TM}$ & \\
\hline 95 & 41 & 42.3 & 41.1 & 42.2 & 41.5 & 42 & 40.2 & 41.3 & 43.6 & 44.9 & \multirow{2}{*}{ Excelente } \\
\hline 90 & 37.1 & 39.1 & 39 & 40.9 & 38.8 & 41 & 39.6 & 40.2 & 41.3 & 42.2 & \\
\hline 75 & 34.8 & 36 & 35.2 & 36.2 & 35.5 & 37.8 & 36.3 & 37.8 & 36 & 37.2 & Bom \\
\hline 50 & 30 & 32.5 & 30.2 & 33 & 31.5 & 33.3 & 30.1 & 32.1 & 32.5 & 33.5 & Média \\
\hline 25 & 27.2 & 30 & 26.9 & 28.8 & 27 & 28.2 & 26 & 27.7 & 24.7 & 26.5 & Regular \\
\hline 10 & 21.3 & 23.9 & 23 & 26.5 & 22 & 24.7 & 21.7 & 23.5 & 20.7 & 22 & \multirow{2}{*}{ Fraco } \\
\hline 5 & 18.9 & 22 & 20.8 & 22.1 & 17.9 & 18.5 & 19 & 20.5 & 18.6 & 20.7 & \\
\hline $\mathrm{N}$ & \multirow{2}{*}{\multicolumn{2}{|c|}{48}} & \multirow{2}{*}{\multicolumn{2}{|c|}{70}} & \multirow{2}{*}{\multicolumn{2}{|c|}{$\begin{array}{c}73 \\
321 \\
\end{array}$}} & \multirow{2}{*}{\multicolumn{2}{|c|}{64}} & \multirow{2}{*}{\multicolumn{2}{|c|}{66}} & \\
\hline Total & & & & & & & & & & & \\
\hline
\end{tabular}

Tabela 4

Valores percentis da flexibilidade (cm) para o teste de sentar e alcançar, convencional (TC) e modificado (TM), na condição com aquecimento para o gênero masculino.

\begin{tabular}{|c|c|c|c|c|c|c|c|}
\hline \multirow{2}{*}{ Percentil } & \multicolumn{2}{|c|}{$18-35$} & \multicolumn{2}{|c|}{$36-50$} & \multicolumn{2}{|c|}{$>50$} & \multirow{2}{*}{ Classificação } \\
\hline & $\mathrm{TC}$ & TM & TC & TM & $\mathrm{TC}$ & TM & \\
\hline 95 & 41.5 & 43.2 & 44.5 & 43.8 & 43.8 & 44.5 & \multirow{2}{*}{ Excelente } \\
\hline 90 & 39 & 41.5 & 37.8 & 38.9 & 37.7 & 40.5 & \\
\hline 75 & 37.1 & 39.5 & 32.7 & 34.8 & 34.3 & 35.6 & Bom \\
\hline 50 & 32.3 & 35 & 27.2 & 30.2 & 28.5 & 30.4 & Média \\
\hline 25 & 28.4 & 30.5 & 23.1 & 25.2 & 23.5 & 25.1 & Regular \\
\hline 10 & 22 & 26 & 19.2 & 21.3 & 17.6 & 20.8 & \multirow{2}{*}{ Fraco } \\
\hline 5 & 18.2 & 23.5 & 16.3 & 17.5 & 15.4 & 18.8 & \\
\hline $\mathrm{N}$ & \multirow{2}{*}{\multicolumn{2}{|c|}{31}} & & & \multirow{2}{*}{\multicolumn{2}{|c|}{34}} & \\
\hline Total & & & \multicolumn{2}{|c|}{91} & & & \\
\hline
\end{tabular}

\section{DISCUSSÃO}

O presente estudo mostra que o Teste de Sentar e Alcançar modificado apresentou melhores resultados quando comparado ao teste convencional, com diferença significativa em ambos os gêneros avaliados. Com relação à condição em que foi realizado o teste (com e sem aquecimento), o protocolo com aquecimento foi superior ao protocolo sem aquecimento, para ambos os gêneros, tanto para o TSA modificado quanto para o TSA convencional.

Nossos achados corroboram com outros estudos que tiveram como objetivo propor estratégias para eliminar as diferenças relacionadas ao comprimento dos membros superiores e inferiores. Hopkins e Hoeger (1992) comparam o TSA original, proposto por Wells e Dillon (1952) com o TSA modificado, proposto por Hopkins (1981) e identificaram diferenças significativas quando um mesmo grupo era submetido em ambos os protocolos. No protocolo utilizado no estudo de Hopkins e Hoeger (1992), a adaptação sugerida foi estabelecer um ponto zero para cada indivíduo, a fim de eliminar as diferenças de comprimentos dos membros superiores, antes da realização do teste. Liemohn et al. (1994) verificaram que a posição do tornozelo (dorsiflexão e flexão-plantar) poderia modificar o score final do teste. A metodologia utilizada foi similar a que foi utilizada no presente estudo (os autores utilizaram homens e mulheres fisicamente ativos). Antes do inicio dos testes os indivíduos realizavam um aquecimento prévio na região lombar, musculatura posterior de coxa e gastrocnêmios. A adaptação sugerida foi realizar o teste em flexão-plantar e não em dorsiflexão, como no teste original. Em ambos os gêneros, o score foi maior quando a flexãoplantar foi utilizada durante o teste, o que corrobora com nossos achados. Quando comparados os testes com e sem aquecimento, o TSA adaptado novamente foi o que apresentou as maiores pontuações. Uma hipótese para essas diferenças seria a ligação entre as fáscias dos gastrocnêmios e dos isquiotibiais, ou a tensão sobre o nervo ciático, que pode restringir o desempenho dos sujeitos quando o tornozelo encontra-se em dorsiflexão 
durante a realização dos testes (Gajdosik, LeVeau, \& Bohannon, 1985).

Em nossos achados quando comparando o TSA realizado com e sem o aquecimento prévio, a realização do aquecimento proporcionou os maiores valores quando comparado sem o aquecimento (tabela 2). Por definição existem dois tipos de aquecimento: o passivo, onde a temperatura muscular e corporal é aumentada por meios externos e, o aquecimento ativo, que é composto basicamente por algum tipo de movimento do corpo, não específico, como por exemplo: caminhar, andar de bicicleta, calistenia, entre outros (Woods et al., 2007). O aquecimento proposto para anteceder o TSA é caracterizado como um aquecimento ativo e, possivelmente, pode proporcionar alterações fisiológicas na musculatura (Woods et al., 2007). Uma hipótese para o aquecimento utilizado no TSA proporcionar os maiores valores, seria recorrente de um aumento da temperatura muscular, que por consequência aumenta a vasodilatação, o que produz um aumento do fluxo sanguíneo na região dos tecidos ativos (musculatura paravertebral, posteriores da perna e coxa), promovendo uma redução da rigidez muscular proporcionando um aumento na viscoelasticidade dos músculos utilizados (Thompson, Ryan, Sobolewski, Conchola, \& Cramer, 2013), proporcionando assim um aumento na amplitude articular, consequentemente um score final maior no TSA.

Comparando entre os gêneros, Liemohn et al. (1994) encontraram diferença com relação as mulheres tanto para o TSA original quanto para o adaptado, o que não foi confirmado em nosso estudo. Uma possível hipótese para esse fato seria as mulheres recrutadas para o estudo de Liemohn et al. (1994) estarem em período fértil do ciclo menstrual, o que resulta em um aumento na liberação de dois hormônios, a relaxina e o estrogênio. A função da relaxina é manter a função por meio do relaxamento de ligamentos, alongamento de fibrocartilagens por atividade colagenolítica e aumento do fluido sinovial na preparação para o parto, ampliando a articulação pélvica. Pode ser ainda que os receptores de estrogênios acumulem relaxina nas articulações pélvicas e cápsula articular (Polden \& Mantle, 1997), o que possivelmente aumentaria a flexibilidade das mulheres no estudo. Tanto em nosso estudo quanto no de Liemohn et al. (1994), não houve qualquer tipo de controle com relação ao ciclo menstrual das mulheres voluntárias.

O TSA é rápido, prático e simples de ser aplicado; qualquer pessoa sem experiência prática consegue utilizá-lo facilmente, além de ser extremamente útil para avaliar uma grande quantidade de indivíduos. Porém, ele apresenta limitações que podem influenciar na pontuação final obtida, consequentemente modificando a classificação do nível de flexibilidade do avaliado. Uma dessas limitações está relacionada à diferença de comprimento dos membros inferiores, e a utilização ou não do aquecimento proposto para o teste.

\section{CONCLUSÕES}

Conclui-se que para avaliar a flexibilidade de membros inferiores, preferencialmente utilizar um TSA adaptado a fim de minimizar uma possível influência do comprimento dos membros inferiores, além da influência do aquecimento proposto para o TSA no valor final do teste.

\section{Agradecimentos:}

Nada a declarar

\section{Conflito de Interesses:}

Nada a declarar

\section{Financiamento: \\ Fundação de Âmparo a Pesquisa do Estado do Amazonas (FAPEAM). Projeto: PIB-S/0025/2010}

\section{REFERÊNCIAS}

ACSM. (2006). Manual do ACSM para avaliação da aptidão física relacionada à saúde ( $1^{\mathrm{a}}$ ed.). São Paulo: Guanabara Koogan. 
Cardoso, J. R., Azevedo, N. C. T., Cassano, C. S., Kawano, M. M., \& Âmbar, G. (2007). Intra and interobserver reliability of angular kinematic analysis of the hip joint during the sit-and-reach test to measure hamstring length in university students. Brazilian Journal of Physical Therapy, 11(2), 133-138. http://doi.org/10.1590/S141335552007000200008

Gajdosik, R. L., LeVeau, B. F., \& Bohannon, R. W. (1985). Effects of ankle dorsiflexion on active and passive unilateral straight leg raising. Physical Therapy, 65(10), 1478-1482.

Garber, C. E., Blissmer, B., Deschenes, M. R., Franklin, B. A., Lamonte, M. J., Lee, I.-M., ... American College of Sports Medicine. (2011). American College of Sports Medicine position stand. Quantity and quality of exercise for developing and maintaining cardiorespiratory, musculoskeletal, and neuromotor fitness in apparently healthy adults: guidance for prescribing exercise. Medicine and Science in Sports and Exercise, 43(7), 1334-1359. http://doi.org/10.1249/MSS.0b013e318213fefb

Hopkins, D. (1981). The relationship between selected anthropometric measures and sit-andreach performance. Comunicação apresentada na American Alliance for Health, Physical Education, Recreation and Dance National Measurement Symposium, Houston, TX.

Hopkins, D. R., \& Hoeger, W. W. K. (1992). A Comparison of the Sit-and-Reach Test and the Modified Sit-and-Reach Test in the Measurement of Flexibility for Males. The Journal of Strength \& Conditioning Research, 6(1), 7-10.

Liemohn, W., Sharpe, G. L., \& Wasserman, J. F. (1994). Criterion Related Validity of the Sitand-Reach Test. The Journal of Strength \& Conditioning Research, 8(2), 91-94.

Mayorga-Vega, D., Merino-Marban, R., \& Viciana, J. (2014). Criterion-Related Validity of Sit-andReach Tests for Estimating Hamstring and Lumbar Extensibility: a Meta-Analysis. Journal of Sports Science \& Medicine, 13(1), 1-14.
Mier, C. M., \& Shapiro, B. S. (2013). Sex differences in pelvic and hip flexibility in men and women matched for sit-and-reach score. Journal of Strength and Conditioning Research / National Strength \& Conditioning Association, 27(4), 1031-1035. http://doi.org/10.1519/JSC.0b013e3182651d7 9

Polden, M., \& Mantle, J. (1997). Fisioterapia em Ginecologia e Obstetrícia (2a ed.). São Paulo: Santos.

Shephard, R. J. (1988). PAR-Q, Canadian Home Fitness Test and exercise screening alternatives. Sports Medicine (Auckland, N.Z.), 5(3), 185195.

Simoneau, G. G. (1998). The Impact of Various Anthropometric and Flexibility Measurements on the Sit-and-Reach Test. The Journal of Strength \& Conditioning Research, 12(4), 232 237.

Thompson, B. J., Ryan, E. D., Sobolewski, E. J., Conchola, E. C., \& Cramer, J. T. (2013). Age related differences in maximal and rapid torque characteristics of the leg extensors and flexors in young, middle-aged and old men. Experimental Gerontology, 48(2), 277-282. http://doi.org/10.1016/j.exger.2012.10.009

Wells, K. F., \& Dillon, E. K. (1952). The Sit and Reach-A Test of Back and Leg Flexibility. Research Quarterly. American Association for Health, Physical Education and Recreation, 23(1), 115-118. http://doi.org/10.1080/10671188.1952.107619 65

Woods, K., Bishop, P., \& Jones, E. (2007). Warmup and stretching in the prevention of muscular injury. Sports Medicine (Auckland, N.Z.), 37(12), 1089-1099.

World Health Organization. (1995). Physical status: the use and interpretation of anthropometry (Technical Report Series No. 854) (pp. 1-452). Genebra: World Health Organization Expert Committee. especificado em contrário e nos conteúdos retirados de outras fontes bibliográficas. 